\title{
Recognition of Isolated Handwritten Kannada Numerals Based on Image Fusion Method
}

\author{
G.G. Rajput and Mallikarjun Hangarge \\ Dept. Of Computer Science, Gulbarga University,Gulbarga \\ ggrajput@yahoo.co.in, mhangarge@yahoo.co.in
}

\begin{abstract}
This paper describes a system for isolated Kannada handwritten numerals recognition using image fusion method. Several digital images corresponding to each handwritten numeral are fused to generate patterns, which are stored in $8 \times 8$ matrices, irrespective of the size of images. The numerals to be recognized are matched using nearest neighbor classifier with each pattern and the best match pattern is considered as the recognized numeral. The experimental results show accuracy of $96.2 \%$ for 500 images, representing the portion of trained data, with the system being trained for 1000 images. The recognition result of $91 \%$ was obtained for 250 test numerals other than the trained images. Further to test the performance of the proposed scheme 4 -fold cross validation has been carried out yielding an accuracy of $89 \%$.
\end{abstract}

\section{Introduction}

Automatic recognition of handwritten digits has been the subject of intensive research during the last few decades. Digit identification is very vital in applications such as interpretation of ID numbers, Vehicle registration numbers, Pin Codes, etc.

Most of the published work has identified a number of approaches for handwritten character and numerals recognition having their own merits and demerits. In most published work, training samples are used for automated digit prototyping which are available as images stimulating direct application of several well investigated image processing techniques [1, 2]. A systematic survey of numerous automated systems can be found in $[3,4]$. An overview of feature extraction methods for offline recognition of isolated characters has been proposed in [5]. A texture based approach using modified invariant moments for pin code script identification is reported in [6]. Unconstrained handwritten character recognition based on fuzzy logic is proposed in [7]. Multiple classifiers based on third order dependency for handwritten numeral recognition is presented in [8]. Unconstrained digit recognition using radon function which represents image as a collection of projections along various directions is reported in [9]. Font and size independent OCR system for printed Kannada documents using support vector machines has been proposed in [10]. Substantial research related to Indian scripts can be found in [11- 15]. From literature survey, it is clear that 
much work has been concentrated for recognition of characters rather than numerals. Moreover, in Indian context, it is evident that still handwritten numerals recognition research is a fascinating area of research to deign a robust optical character recognition (OCR), in particular for handwritten Kannada numeral recognition. Hence, we are motivated to design a simple and robust algorithm for handwritten Kannada numerals recognition system. In this paper, we propose a simple and effective method for feature extraction based on image fusion technique [16]. The 64 dimensional features are used to classify the handwritten Kannada numerals using basic nearest neighbour classifier and aimed to obtain encouraging results. The rest of the paper is organized as follows.

In Section 2, the brief overview of data collection and pre-processing is presented. Section 3 deals with the feature extraction and recognition system. The algorithm is presented in Section 4. The experimental results obtained are presented in Section 5. Conclusion is given in Section 6.

\section{Data Collection and Preprocessing}

The Kannada language is one of the four major south Indian languages. The Kannada alphabet consists of 16 vowels and 36 consonants. Vowels and consonants are combined to form composite letters. Writing style in the script is from left to right. It also includes 10 different symbols representing the ten numerals of the decimal number system shown in Fig. 1.

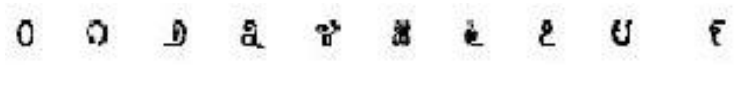

Fig. 1. Kannada numerals 0 to 9

The database of totally unconstrained Kannada handwritten numerals has been created for validating the recognition system, as the standard database is not available at the moment. Hence, samples of 100 writers were chosen at random from schools, colleges and professionals for collecting the handwritten numerals. The writers were not imposed by any constraint like type of pen and style of writing etc., and the purpose of data collection is not disclosed. Writers were provided with plain A4 normal papers and were asked to write Kannada numerals 0 to 9.

The collected documents are scanned using HP-Scan jet 2400 scanner at 300 dpi which usually yields a low noise and good quality document image. The digitized images are stored as binary images in bit map format. The noise has been removed using morphological dilate and erode operations. Further, it is assumed that the skew correction has been performed before pre-processing. The preprocessed image is inverted resulting in the background as black (binary 0) and numeral as white(binary 1). A total of 1000 binary images of handwritten Kannada numerals are obtained. A sample of Kannada handwritten numerals from this data set is shown in the Fig. 2. 


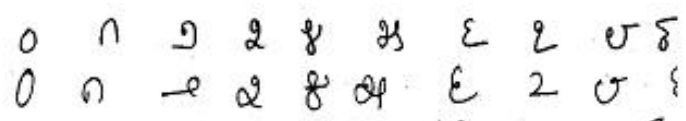 \\ $0 \cap \rightarrow 282 \varepsilon 20\}$}

Fig. 2. Sample handwritten Kannada numerals 0 to 9

\section{Features Extraction and Recognition}

\subsection{Feature Extraction}

Region labeling is performed on the preprocessed image and a minimum rectangle bounding box is inserted over the numeral and the numeral is cropped. The normalization of the numerals is essential because of the varied writing styles of the writers in the shapes and sizes. Therefore, to bring uniformity among the input numerals, the cropped numeral is normalized to fit into a size of $32 \times 32$ pixels without disturbing the aspect ratio. (See Fig. 3(b)). Next, image thinning is performed on this numeral. The purpose of thinning is to reduce the image components to their essential information so that further analysis and recognition are facilitated. It is very important to extract features in such a way that the recognition of different numerals becomes easier on the basis of individual features of each numeral. Therefore, the binary image is divided into 64 zones of equal size, each zone being of size $4 \times 4$ pixels, so that the portions of numeral will lie in some of these zones. Obviously, there could be zones that are empty. A pattern matrix $M p t_{j}$ of size $8 \times 8$ is created, where $\mathrm{j}$ ranges from 0 to 9 representing the numerals. Next, for each zone of the image, if the number of on pixels is greater than $5 \%$ of total pixels in that zone, 1 is stored in $M p t_{j}$, for that image. Thus, Mptj represents the reduced image (see Fig. 3 (c)) having the same features as that of its original image. The reduced images, $M p t_{j}$ 's, of each numeral are fused to generate the pattern matrix , $P_{M j}$, of size $8 \mathrm{x} 8$, j ranging from 0 to 9 , using the following recursive equation [16].

$$
P_{M j}^{N e w}=\frac{1}{n u m+2}\left(n u m * P_{M j}^{O l d}+M p t_{j}\right), \quad 0 \leq j \leq 9
$$

Here, $P_{M j}^{N e w}$ is the fused pattern matrix obtained after fusing training images contained in $M p t_{j}$ and $P_{M j}^{O l d}$ (already stored in the pattern matrix table). $P_{M j}^{N e w}$ is copied back to the table along with num increased by 1 . The content of each cell of fused pattern matrix represents the probability of occurrence of a white pixel that is mapped with the test image to a typical numeral.

\subsection{Recognition}

We use nearest neighbour classifier for recognition purpose. The test numeral feature vector is classified to a class, to which its nearest neighbour belongs to. 


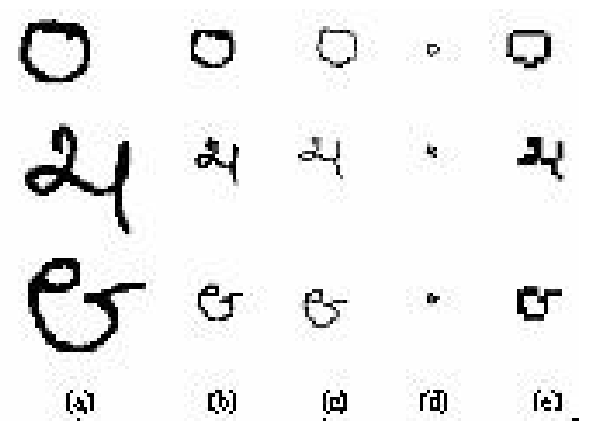

Fig. 3. (a) Original Image, (b) Normalized image, (c) Thinned image,(d) Reduced image, (e) Enlarged view of reduced image

Feature vectors stored priori are used to decide the nearest neighbour of the given feature vector. The recognition process is described below.

The extracted features form the knowledge base and are used to identify the test numerals. The test numeral which is to be recognized is processed in a similar way up to the image reduction step to generate the matrix $M p_{\text {test }}$. Euclidean distance between $M p_{\text {test }}$ and, j ranging from 0 to 9 , is computed using the formula given below.

$$
D_{j}(x)=\left\|M p_{\text {test }}-P_{M j}\right\| \quad 0 \leq j \leq 9
$$

where $M p_{\text {test }}$ represents the pattern matrix of input test image and $P_{M j}$ is the fused pattern matrix of jth numeral in the database. The nearest neighbour technique assigns the test numeral to a class that has the minimum distance. The corresponding numeral is declared as recognized numeral.

\section{Algorithm}

Feature extraction algorithm is given below:

Input:Digitized binary Kannada numeral image set Output:Pattern matrix of size $8 \times 8$ as feature vector Method:Feature Extraction

Step 1: For each sample of Kannada numeral do

i remove noise, if any

ii invert the image to get background black and numeral white

iii fit a bounding box for the numeral and crop the numeral

iv resize the cropped numeral to $32 \times 32$ pixels without loosing aspect ration

$\mathbf{v}$ apply thinning process to the normalized image

vi divide the normalized image into 64 zones of equal size

vii create a pattern matrix $M p_{t j}$ of size $8 \times 8$ 
viii For each zone if the number of on pixels is greater than $5 \%$ of total pixels store 1 in $M p_{t j}$

ix Fuse the reduced image $M p_{t j}$ with $P_{M j}$ using equation (1) and store back the result. $P_{M j}$ forms the feature matrix for that numeral

Step2. Repeat step 1 for all numerals.

Recognition algorithm is given below.

Input: Digitized binary Kannada numeral test image

Output: recognized numeral display

Method: Recognition

Step 1: For the test Kannada numeral perform feature extraction to obtain pattern matrix $M p_{\text {test }}$ of size $8 \times 8$.

Step 2: Compute the Euclidian distance between the test pattern $M p_{\text {test }}$ and each of the trained patterns $P_{M j}$ using equation (2).

Step 3: Choose the minimum distance as the best match and declare the corresponding numeral as the recognized numeral.

The proposed image recognition system was implemented with Matlab scripts as illustrated in Fig 4.

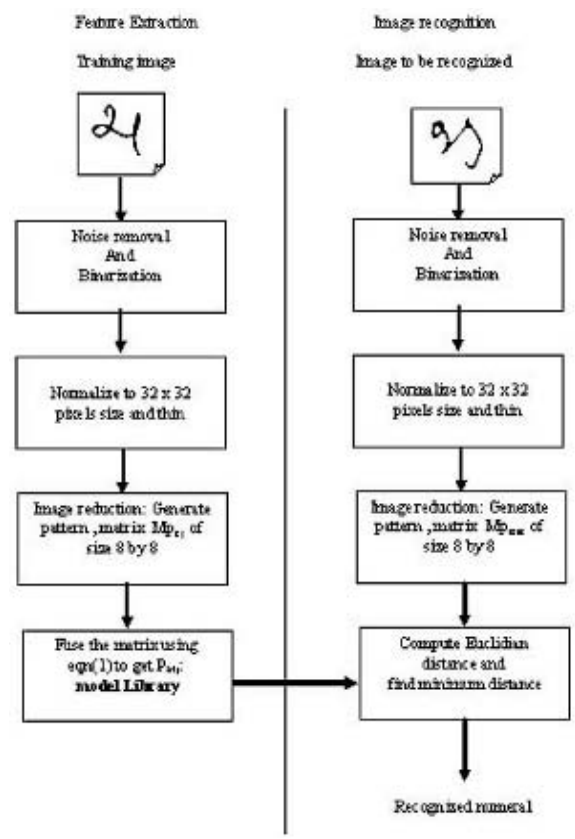

Fig. 4. Recognition system 


\section{Results and Discussion}

The experiments were carried out on the data set discussed in section 2 . To see how well the system represents the data it has been trained on, we extracted 500 numerals at random, 50 samples for each numeral, and checked the results. Further, the test data contained a separate set of 250 numerals other than the training set. The Table 1 shows the recognition results for both the cases.

Table 1. Experimental results

\begin{tabular}{|c|c|c|c|c|}
\hline \multirow{2}{*}{ Digits } & \multicolumn{2}{|c|}{$\begin{array}{c}\text { For a portion of trained } \\
\text { data }\end{array}$} & \multicolumn{2}{c|}{ For test data } \\
\cline { 2 - 5 } & $\begin{array}{c}\text { No. of trained } \\
\text { numerals }\end{array}$ & $\begin{array}{c}\% \text { of } \\
\text { recognition }\end{array}$ & $\begin{array}{c}\text { No. of test } \\
\text { numerals }\end{array}$ & $\begin{array}{c}\% \text { of } \\
\text { recognition }\end{array}$ \\
\hline 0 & 50 & 100 & 25 & 96 \\
\hline 0 & 50 & 86 & 25 & 100 \\
\hline 5 & 50 & 100 & 25 & 100 \\
\hline 2 & 50 & 96 & 25 & 72 \\
\hline 8 & 50 & 96 & 25 & 92 \\
\hline 29 & 50 & 92 & 25 & 92 \\
\hline$\varepsilon$ & 50 & 98 & 25 & 76 \\
\hline 2 & 50 & 98 & 25 & 92 \\
\hline $\mathcal{E}$ & 50 & 98 & 25 & 100 \\
\hline $\mathcal{E}$ & 50 & 98 & 25 & 92 \\
\hline \multicolumn{2}{|c|}{ Average recognition } & $96.2 \%$ & & $91.2 \%$ \\
\hline
\end{tabular}

I $\mathrm{n}$ the test set, a recognition rate of $91.2 \%$ was achieved. Understandably, the training set produced much higher recognition rate of $96.2 \%$ than the test data. Further, to validate the performance of the system trained for 1000 images, we carried out K-Fold Cross-validation on 1000 images. With $\mathrm{K}=4$, for each of $\mathrm{K}$ experiments we used K-1 folds for training and the remaining one for testing. The recognition rate turned out to be $89 \%$.

It is a difficult task to compare results for handwritten Kannada numeral recognition with other researchers in the literature due the differences in experimental methodology, experimental settings and the size of the database used. Table 2 presents the comparison of the proposed method with other methods in

Table 2. Comparative results

\begin{tabular}{|l|l|l|l|l|}
\hline \multicolumn{1}{|c|}{ Method } & $\begin{array}{l}\text { Feature extraction } \\
\text { method }\end{array}$ & $\begin{array}{l}\text { Data set } \\
\text { size }\end{array}$ & Classifier & $\begin{array}{l}\text { Success } \\
\text { rate }\end{array}$ \\
\hline $\begin{array}{l}\text { V. N. Manjunath } \\
\text { Aradhya et. Al.[9] }\end{array}$ & Radon transform & 1000 & $\begin{array}{l}\text { Nearest } \\
\text { Neighbour }\end{array}$ & 91.2 \\
\hline N. Sharma et. Al. [11] & chain code & 2300 & $\begin{array}{l}\text { quadratic } \\
\text { classifier }\end{array}$ & 97.87 \\
\hline $\begin{array}{l}\text { Dinesh Acharya U. et } \\
\text { Al. [15 ] }\end{array}$ & Structural features & 500 & $\begin{array}{l}\text { k-means } \\
\text { classifier }\end{array}$ & 90.5 \\
\hline Proposed & Image Fusion & 1000 & $\begin{array}{l}\text { Nearest } \\
\text { Neighbour }\end{array}$ & 91.2 \\
\hline
\end{tabular}


the literature dealing with Kannada numerals. The proposed method yields a comparable recognition rate of $91.2 \%$ at lesser computation cost even with the basic classifier. However, the higher recognition rate can certainly be achieved by using better classifiers.

\section{Conclusion}

A system capable of recognizing isolated handwritten Kannada numerals is presented. Image fusion method is used to generate patterns as feature matrices of 64 dimensions. The nearest neighbor classification is used to identify the test numeral. The novelty of this paper is that the proposed scheme is simple and effective. The results are discussed for portion of the trained data as well as for unseen instances of data collected from the writers other than that for trained data. The recognition rates are encouraging and supported by 4-Fold cross validation. Our future aim is to improve the recognition rate using fuzzy based classifier and to perform experiments using larger data set. Further, the proposed scheme may be tested for recognition of handwritten numerals and characters of other languages also.

Acknowledgement. The authors thank the referees for their comments and suggestions. The authors wish to acknowledge Dr. P. S. Hiremath, Dr. B. V. Dhandra, Department of Computer Science, Gulbarga University, Gulbarga and Dr. P. Nagabhushan, Dr. Hemantkumar and Dr. Guru, Department of Computer Science, University of Mysore, Mysore for their support.

\section{References}

1. Duda, R., Hart, P.: Pattern Classification and Scene Analysis. John Wiley, New York (1973)

2. Gader, P., Khabou, M.: Automated Feature Generation for Handwritten Digit Recognition. IEEE Transactions On Pattern Analysis And Machine Intelligence 18(12), 1256-1261 (1996)

3. Casy, R., Lecolinet, E.: A Survey Of Methods And Strategies In Character Segmentation. IEEE Transactions On Pattern Analysis And Machine Intelligence 18(7), 690-706 (1996)

4. Plamodin, R., Srihari, S.N.: Online and Offline Handwriting Recognition: A Comprehensive survey. IEEE Transaction on Pattern Analysis and Machine Intelligence 22(1), 63-84 (2000)

5. Trier, O., Jain, A.K., Taxt, T.: Feature Extraction Methods For Character Recognition - A Survey Pattern Recognition. 29(4), 641-662 (1996)

6. Nagabhushan, P., Angadi, S.A., Anami, B.S.: An Intelligent Pin Code Script Identification Methodology Based On Texture Analysis using Modified Invariant Moments. In: Proc. of ICCR, pp. 615-623. Allied Pub (2005)

7. Hanmandlu, M., Mohan, R.M., Chakraborty, S., Goyal, S., Choudhary, D.R.: Unconstrained Handwritten Character Recognition Based on Fuzzy Logic. Pattern Recognition 36, 603-623 (2003) 
8. Kang, H.J.: Combining Multiple Classifiers Based on Third Order Dependency For Handwritten Numeral Recognition. Pattern Recognition Letters 24, 3027-3036 (2003)

9. Aradhya, V.N.M., Kumar, G.H., Noushath, S.: Robust Unconstrained Handwritten Digit Recognition Using Radon Transform. In: Proc. of IEEE-ICSCN 2007, IEEE Computer Society Press, Los Alamitos (2007)

10. Ashvin, T.V., Shastry, P.S.: A Font And Size Independent Ocr System For Printed Kannada Documents using Support Vector Machines. Sadhana 27(1), 23-24 (2002)

11. Sharma, N., Pal, U., Kimura, F.: Recognition of Handwritten Kannada Numerals. In: Proc. of 9th International Conference on Information Technology (ICIT 2006), pp. 133-136 (2006)

12. Hariharana, S.: Recognition of Handwritten Numerals Through Genetic Based Machine Learning. Jour. of the CSI 30(2), 16-22 (2000)

13. Bhattacharya, U., et al.: Recognition of Hand Printed Bangla Numerals Using Neural Network Models. In: Pal, N.R., Sugeno, M. (eds.) AFSS 2002. LNCS (LNAI), vol. 2275, pp. 228-235. Springer, Heidelberg (2002)

14. Raju, G.: Recognition Of Unconstrained Handwritten Malayalam Characters Using Zero Crossing of Wavelet Coefficients. In: Proc. of ADCOM, pp. 217-221 (2006)

15. Dinesh Acharya, U., Subbareddy, N.V., Krishnamoorthy,: Isolated Kannada Numeral Recognition Using Structural Features and K-Means Cluster. In: Proc. of IISN-2007, pp. 125-129 (2007)

16. Chakraborty, R., Sil, J.: Handwritten Character Recognition Systems Using Image Fusion And Fuzzy Logic. In: PReMI 2005. LNCS, vol. 3766, pp. 344-349. Springer, Heidelberg (2005) 